\title{
Article
}

\section{The Antidiabetic Agent Metformin Inhibits IL-23 Production in Murine Bone-Marrow-Derived Dendritic Cells}

\author{
Tomoyo Matsuda-Taniguchi ${ }^{1}$, Masaki Takemura ${ }^{1}$ (D), Takeshi Nakahara ${ }^{1}$, Akiko Hashimoto-Hachiya ${ }^{1}$, \\ Ayako Takai-Yumine ${ }^{1}$, Masutaka Furue ${ }^{1}$ (D) and Gaku Tsuji 1,2,*(D) \\ 1 Department of Dermatology, Graduate School of Medical Sciences, Kyushu University, \\ Fukuoka 812-8582, Japan; taniguchi.tomoyo.735@s.kyushu-u.ac.jp (T.M.-T.); \\ take0917@dermatol.med.kyushu-u.ac.jp (M.T.); nakahara.takeshi.930@m.kyushu-u.ac.jp (T.N.); \\ ahachi@dermatol.med.kyushu-u.ac.jp (A.H.-H.); a-takai@med.kyushu-u.ac.jp (A.T.-Y.); \\ furue@dermatol.med.kyushu-u.ac.jp (M.F.) \\ 2 Research and Clinical Center for Yusho and Dioxin, Kyushu University Hospital, Fukuoka 812-8582, Japan \\ * Correspondence: gakku@dermatol.med.kyushu-u.ac.jp; Tel.: +81-92-642-5585
}

\section{check for} updates

Citation: Matsuda-Taniguchi, T.; Takemura, M.; Nakahara, T.; Hashimoto-Hachiya, A.; Takai-Yumine, A.; Furue, M.; Tsuji, G. The Antidiabetic Agent Metformin Inhibits IL-23 Production in Murine Bone-Marrow-Derived Dendritic Cells. J. Clin. Med. 2021, 10, 5610. https:// doi.org/10.3390/jcm10235610

Academic Editor:

Francesco Lacarrubba

Received: 25 October 2021

Accepted: 29 November 2021

Published: 29 November 2021

Publisher's Note: MDPI stays neutral with regard to jurisdictional claims in published maps and institutional affiliations.

Copyright: (C) 2021 by the authors. Licensee MDPI, Basel, Switzerland. This article is an open access article distributed under the terms and conditions of the Creative Commons Attribution (CC BY) license (https:// creativecommons.org/licenses/by/ $4.0 /)$.

\begin{abstract}
Psoriasis is a chronic inflammatory skin disease, and its immune mechanism has been profoundly elucidated. Biologics targeting interleukin (IL)-23 have prevented the development of psoriasis. As major sources of IL-23, dendritic cells (DCs) play a pivotal role in psoriasis; however, the regulatory mechanism of IL-23 in DCs remains unclear. IL-36 $\gamma$ was reported to reflect the disease activity of psoriasis. Therefore, we hypothesized that IL-36 $\gamma$ may affect IL-23 production in DCs. To reveal the mechanism by which IL-36 $\gamma$ controls IL-23 production in DCs, we analyzed murine bone marrow-derived DCs (BMDCs) stimulated with IL-36 $\gamma$. IL-36 $\gamma$ stimulation upregulated the mRNA and protein expression of Nfkbiz in BMDCs. Nfkbiz knockdown using siRNA transfection partially inhibited the upregulation of IL-23 mRNA expression induced by IL-36 $\gamma$ stimulation. Since NF- $\kappa B$ signaling regulates Nfkbiz expression and the anti-diabetic agent metformin reportedly modulates NF- $\kappa B$ signaling, we examined the effect of metformin treatment on IL-36 $\gamma$-induced IL-23 production.

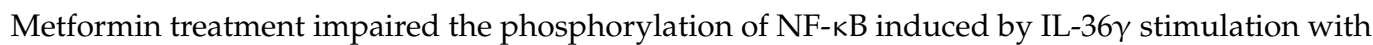
the subsequent downregulation of Nfkbiz, resulting in the inhibition of IL-23 production in BMDCs. These data provided evidence that metformin treatment can inhibit IL-36 $\gamma$-mediated IL-23 production in BMDCs, which might contribute to the prevention of psoriasis.
\end{abstract}

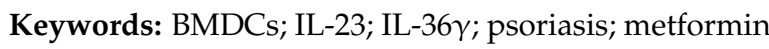

\section{Introduction}

Psoriasis is an immune-mediated inflammatory skin disease affecting $2-4 \%$ of the global population [1]. The skin lesions in psoriasis manifest desquamative erythema, which profoundly impairs patients' quality of the life [2]. The pathology of psoriasis is characterized by epidermal hyperproliferation, the intraepidermal accumulation of neutrophils, and the infiltration of dermal inflammatory cells such as T-cells, macrophages, and dendritic cells (DCs) [3]. Among these immune cells, DC counts are increased significantly in psoriatic lesions [4]. Furthermore, autoantigens from keratinocytes activate plasmacytoid DCs (pDCs) in the dermis. pDCs produce type I interferon and tumor necrosis factor- $\alpha$, which activates classical DCs (cDCs), resulting in interleukin (IL)-23 secretion. IL-23 is mostly produced by cDCs, which correspond to CD1c+ DCs in humans. IL-23 by cDCs promotes Th17 differentiation in mice and humans [5]. In a murine model of psoriasis induced by topical imiquimod (IMQ), DCs were identified as the major source of IMQ-induced IL-23, which is critical for the development of psoriatic skin lesions [6,7]. Several reports have shown that IL-23 derived from DCs is involved in the pathogenesis of psoriasis [8-10]. Therefore, IL-23-producing DCs play a central role in the pathogenesis of psoriasis, which supports clinical evidence that the administration of monoclonal antibodies against IL-23 
such as guselkmab, rizankizumab, and tildrakizumab can facilitate the achievement of a Psoriasis Area and Severity Index 90 response at week 16 (67-75\%) [11-13]. Whereas IL-23 is a key regulator of IL-17 production, the mechanism of IL-23 production by DCs in psoriasis remains unclear. Recently, serum levels of IL-36 $\gamma$, a member of the IL-36 family, were identified as a disease activity marker of psoriasis [14]. Meanwhile, IL-36 $\gamma$ is highly expressed in the epidermis in psoriatic lesions [15]. As IL-36 $\gamma$ derived from keratinocytes potentially activates DCs [16], we hypothesized that IL-36 $\gamma$ is involved in IL-23 production in DCs during the pathogenesis of psoriasis. To test this hypothesis, we analyzed murine bone marrow-derived DCs (BMDCs) stimulated with IL-36 $\gamma$.

Furthermore, we examined whether metformin, an antidiabetic agent, modulates IL-36 $\gamma$ signaling in BMDCs. Metformin is mostly used to treat type 2 diabetes (T2DM), and a high prevalence of T2DM in patients with severe psoriasis has been identified [17]. In clinical studies of patients with psoriasis, long-term treatment with metformin has been shown to reduce the risk of psoriasis [18]. In addition, metformin administration has been shown to improve the severity of psoriasis $[19,20]$. These clinical results support the likelihood that metformin treatment is effective against both psoriasis and T2DM; however, the molecular mechanism remains unknown.

\section{Materials and Methods}

\subsection{Reagents and Antibodies}

Anti-murine NF-кB p65 monoclonal rabbit antibody (Abcam, Cambridge, UK), antimurine NF-kB p65 (phospho Ser536) polyclonal rabbit antibody (Abcam), anti-murine $\mathrm{I} \kappa \mathrm{B} \zeta$ (protein corded by NFKBIZ gene) polyclonal rabbit antibody, and anti-murine $\beta$-actin monoclonal mouse antibody (Cell Signaling Technology, Danvers, MA, USA) were used for Western blotting. Dimethyl Sulfoxide (DMSO) was purchased from Nacalai Tesque, Inc. (Kyoto, Japan). Metformin hydrochloride and BAY 11-7082 were obtained from Tokyo Chemical Industry Co., Ltd. (Tokyo, Japan). Murine recombinant IL-36 $\gamma$ was obtained from R\&D Systems (Minneapolis, MN, USA).

\subsection{Generation of BMDCs and Cell Culture}

C57BL/6N female mice were housed in a clean facility until 6 weeks of age by CLEA Japan, Inc. (Fujinomiya, Japan). The animal experiments were conducted in accordance with a protocol reviewed and approved by the animal facility center of Kyushu University (A21-283-0, 2021-2023). Bone marrow cells freshly isolated from the femoral and tibial bones of mice were cultured in RPMI 1640 medium (Merck KGaA, Darmstadt, Germany) containing $1 \mathrm{mmol} / \mathrm{L}$ sodium pyruvate (Thermo Fisher Scientific, Waltham, MA, USA), $10 \mathrm{mmol} / \mathrm{L}$ 4-(2-hydroxyethyl)-1-piperazineethanesulfonic acid (Thermo Fisher Scientific), 1\% Minimum Essential Medium Non-Essential Amino Acids (Thermo Fisher Scientific), 10\% FBS (Capricorn Scientific GmbH, Ebsdorfergrund, Germany), 50 nmol/L $\beta$-mercaptoethanol (Nacalai Tesque), and antibiotic-antimycotic $100 \times(100 \mathrm{U} / \mathrm{mL}$ penicillin, $100 \mathrm{mg} / \mathrm{mL}$ streptomycin, and $0.25 \mu \mathrm{g} / \mathrm{mL}$ amphotericin B; Thermo Fisher Scientific) containing GM-CSF (10 ng/mL) (PeproTech, Cranbury, NJ, USA). On day 3, half of the culture medium and GM-CSF were added. On day 5, non-adherent cells were subcultured, and GM-CSF was added. On day 7, half of the culture medium and GM-CSF were added. On day 9, non-adherent cells were harvested. These cells were purified immunomagnetically via three rounds of positive selection with CD11c (N418) MicroBeads (Miltenyi Biotec, Bergisch Gladbach, Germany). Purified BMDCs were cultured with/without stimulants such as IL-36 $\gamma$, metformin, and BAY 11-7082 for the indicated times. Culture supernatant was collected after $24 \mathrm{~h}$ and analyzed by ELISA. Cells were also collected for quantitative reverse transcription (qRT)-PCR or Western blotting.

\subsection{Transfection of Small Interfering RNAs (siRNAs) against Nfkbiz}

siRNAs against Nfkbiz and non-targeting siRNA (control siRNA) were obtained from Thermo Fisher Scientific. Cells were incubated in culture medium with a mixture 
containing $300 \mathrm{nM}$ siRNA for transfection using program DK-100 following the Amaxa ${ }^{\circledR}$ 4 D-Nucleofector ${ }^{\circledR}$ Protocol for Immature Mouse Dendritic Cells For 4 D-Nucleofector ${ }^{\circledR} X$ Unit (Lonza Group AG, Basel, Switzerland).

\section{4. $q R T-P C R$}

Total RNA was extracted using an RNeasy ${ }^{\circledR}$ Mini kit (Qiagen, Venlo, The Netherlands). Reverse transcription was performed using a PrimeScript ${ }^{\mathrm{TM}}$ RT reagent kit (Takara Bio, Shiga, Japan). qRT-PCR was conducted on a CFX Connect ${ }^{\mathrm{TM}}$ Real-time System (Bio-Rad, Hercules, CA, USA). Gene expression levels of IL-23 and Nfkbiz were determined by qRTPCR using TaqMan Fast Advanced Master Mix (Thermo Fisher Scientific). Amplification was initiated at $95^{\circ} \mathrm{C}$ for $20 \mathrm{~s}$ as the first step, followed by 40 cycles of qRT-PCR at $95^{\circ} \mathrm{C}$ for $3 \mathrm{~s}$ and at $60^{\circ} \mathrm{C}$ for $30 \mathrm{~s}$ as the second step. mRNA expression was measured in triplicate with normalization by the housekeeping gene Ywhaz, and expression was indicated as the fold change relative to the control group. Primer sequences are listed in Table S1.

\subsection{Western Blotting}

Cells were incubated for $5 \mathrm{~min}$ in cOmplete ${ }^{\mathrm{TM}}$ Lysis-M (Roche Diagnostics, Basel, Switzerland). The protein concentration in the lysate was measured using a BCA Protein Assay Kit (Thermo Fisher Scientific). Equal amounts of protein $(15 \mu \mathrm{g})$ were dissolved in Bolt LDS sample buffer (Thermo Fisher Scientific) and a 10\% sample reducing agent (Thermo Fisher Scientific). The lysates were boiled at $70{ }^{\circ} \mathrm{C}$ for $10 \mathrm{~min}$ and then to electrophoresis in NuPAGE 4-12\% Bis-Tris gels (Thermo Fisher Scientific) at $200 \mathrm{~V}$ for $25 \mathrm{~min}$. The proteins were then transferred onto polyvinylidene difluoride membranes (Thermo Fisher Scientific), which were blocked with WesternBreeze Blocker/Diluent (Thermo Fisher Scientific). The membranes were then probed with anti-murine NF- $\mathrm{kB}$ p65 monoclonal rabbit antibody, anti-murine NF-kB p65 (phospho Ser536) polyclonal rabbit antibody, and anti-murine I $\mathrm{\kappa} B \zeta$ p polyclonal rabbit antibody (all from Cell Signaling Technology) overnight at $4{ }^{\circ} \mathrm{C}$. Horseradish peroxidase-conjugated anti-rabbit IgG antibodies (Cell Signaling Technology) served as secondary antibodies. Protein bands were visualized with ChemiLumi One Super (Nacalai Tesque) using the ChemiDoc touch imaging system (Bio-Rad). The membranes were then re-blotted with Restore ${ }^{\mathrm{TM}}$ PLUS Western Blot Stripping Buffer (Thermo Fisher Scientific) and anti-murine $\beta$-actin mouse antibody $30 \mathrm{~min}$ at room temperature. Horseradish peroxidase-conjugated anti-mouse IgG antibodies served as secondary antibodies. Protein bands were visualized with SuperSignal ${ }^{\mathrm{TM}}$ West Pico PLUS Chemiluminescent Substrate (Thermo Fisher Scientific) using the ChemiDoc touch imaging system (Bio-Rad). Densitometric analysis of the bands was performed using ImageJ software. ImageJ is a public domain, Java-based image processing program developed at the $\mathrm{Na}-$ tional Institutes of Health (Bethesda, MD, USA). Experiments were repeated three times in separate experiments.

\subsection{ELISA}

A murine IL-23 ELISA Kit (R\&D Systems) was used for ELISA in accordance with the manufacturer's protocol. Optical density was measured using a DTX 800 Multimode Detector (Beckman Coulter, Brea, CA, USA).

\subsection{Statistical Analysis}

Statistical analysis was performed with GraphPad Prism 5.0 (GraphPad Software, Inc., La Jolla, CA, USA). An unpaired Student's $t$-test was used to analyze the results, and a $p$-value of less than 0.05 was considered statistically significant.

\section{Results}

\subsection{IL-36 $\gamma$ Stimulation Upregulated IL-23 and Nfkbiz Expression in BMDCs}

To investigate the mechanism by which IL-36 $\gamma$ regulates IL-23 expression in DCs, we

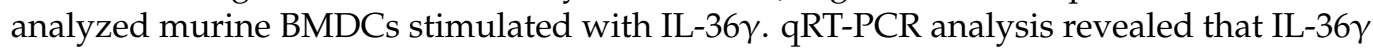


stimulation (100 ng/mL) for 1, 2, 4, or $6 \mathrm{~h}$ upregulated IL-23 mRNA expression with expres-

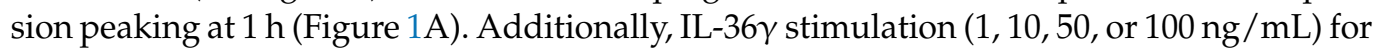
$1 \mathrm{~h}$ upregulated IL-23 mRNA expression in a concentration-dependent manner (Figure 1B). Furthermore, ELISA of the culture medium of BMDCs stimulated with IL-36 $\gamma(1,10,50$, or $100 \mathrm{ng} / \mathrm{mL}$ ) for $24 \mathrm{~h}$ revealed IL-23 production in a concentration-dependent manner (Figure 1C). We measured IL-23 production by ELISA following stimulation for 1 or $6 \mathrm{~h}$; however, IL-23 was undetectable (data not shown). We believe that $24 \mathrm{~h}$ are required for IL-23 secretion to proceed after IL-23 mRNA expression is increased. As NFKBIZ is reported to be a key transcriptional regulator of IL-36-related gene expression in human psoriatic keratinocytes [21,22], we evaluated Nfkbiz expression in addition to IL-23 expression in murine BMDCs. qRT-PCR analysis illustrated that IL-36 $\gamma$ stimulation upregulated Nfkbiz mRNA expression (Figure 1D), which was in a concentration-dependent manner (Figure 1E). Western blotting analysis confirmed that IL-36 $\gamma$ stimulation $(100 \mathrm{ng} / \mathrm{mL})$ for $1,2,4$, or $6 \mathrm{~h}$ upregulated IKB $\zeta$ (protein corded by Nfkbiz gene) protein expression (Figure 1F).
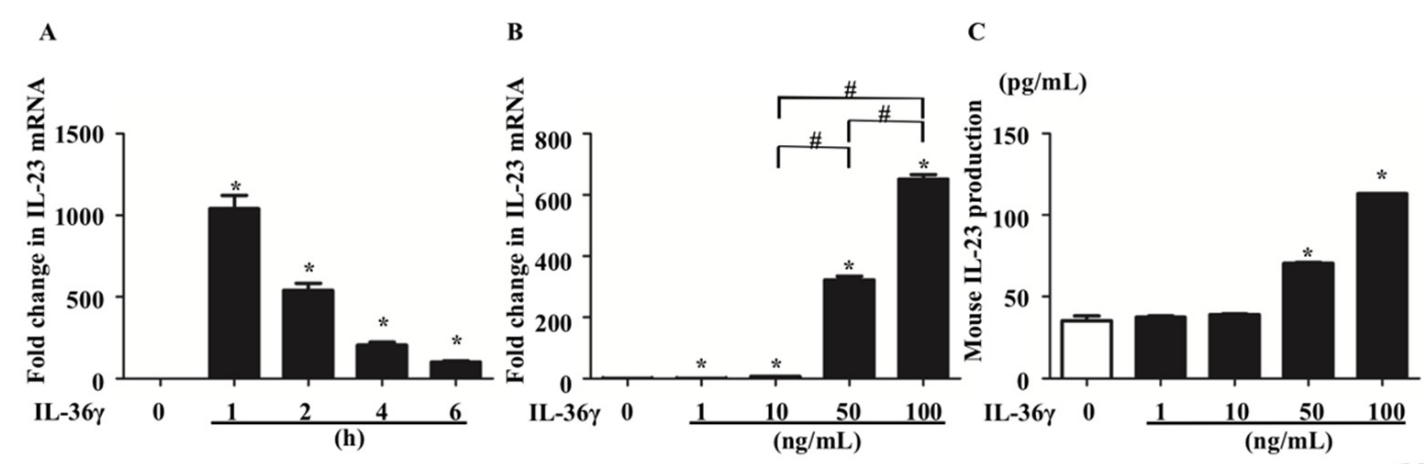

D

E
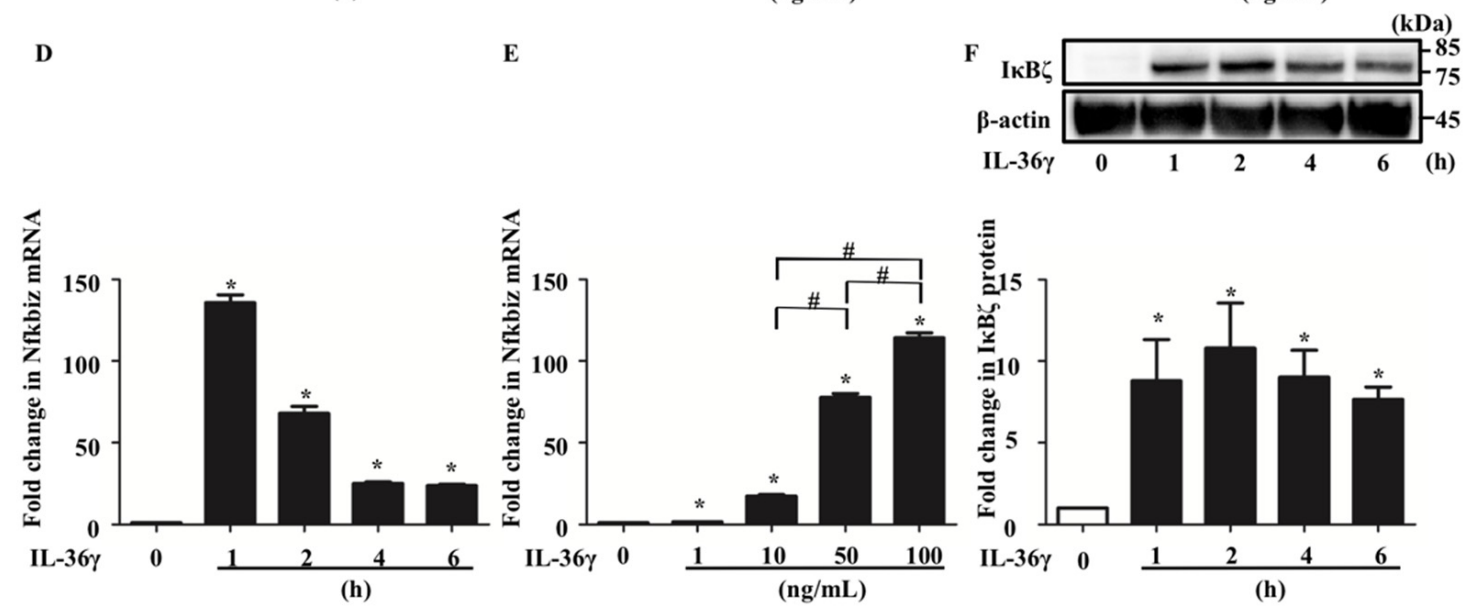

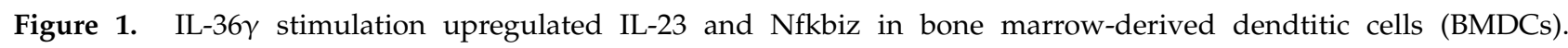

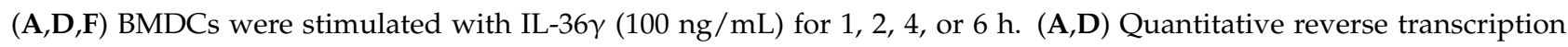
(qRT)-PCR. (F) Western blotting. IкB $\zeta$ protein levels are normalized to $\beta$-actin protein levels using ImageJ and expressed as

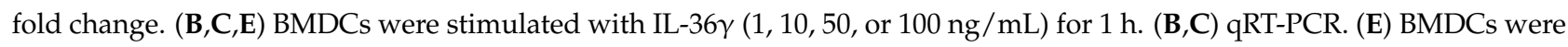

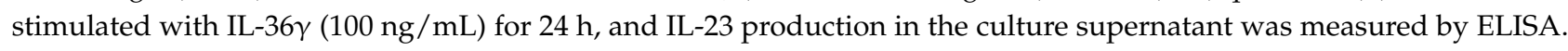
(A-D) Data are expressed as the mean \pm standard error of the mean (SEM); $n=3$ /group. * Significant differences between the IL-36 $\gamma$-stimulated groups and control groups $(p<0.05)$. \# Significant differences between the IL-36 $\gamma$-stimulated groups of each dose $(p<0.05)$. mRNA levels normalized for Ywhaz expression were expressed as the fold change compared to that in the control group. (C) Data are expressed as the mean \pm SEM; $n=3$ /group; * $p<0.05$. (F) Data are representative of experiments repeated three times with similar results. 


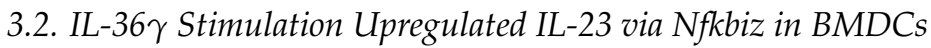

Next, we examined whether Nfkbiz is involved in IL-23 upregulation induced by IL-36 $\gamma$ in murine BMDCs. We transfected BMDCs with either scrambled siRNA (si-control)

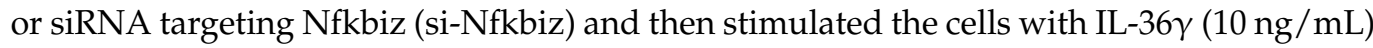
for $1 \mathrm{~h}$. Although the transfection of si-Nfkbiz alone did not alter mRNA and I $\mathrm{KB} \zeta$ protein expression in BMDCs, it successfully downregulated Nfkbiz mRNA (Figure 2A) and protein expression (Figure 2B) in BMDCs stimulated with IL-36 $\gamma$. This finding may be related to the partial depletion of the target gene because siRNA transfection is difficult in DCs [23]. Furthermore, we observed that depletion of Nfkbiz via siRNA transfection partially canceled IL-36 $\gamma$ stimulation-induced IL-23 mRNA upregulation (Figure 2C). Although we attempted to measure IL-23 production in the culture supernatant of siRNAtransfected BMDCs using ELISA, we could not detect IL-23 production, which may be attributable to cell damage caused by the siRNA transfection procedure. These results

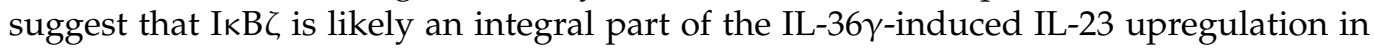
murine BMDCs.

A

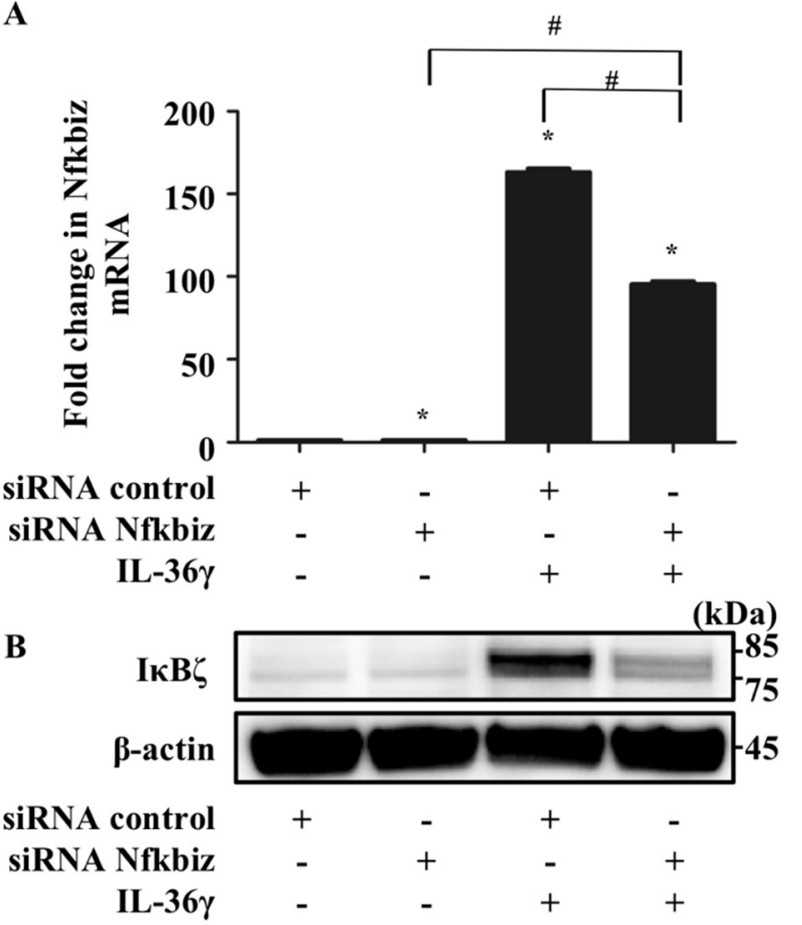

C

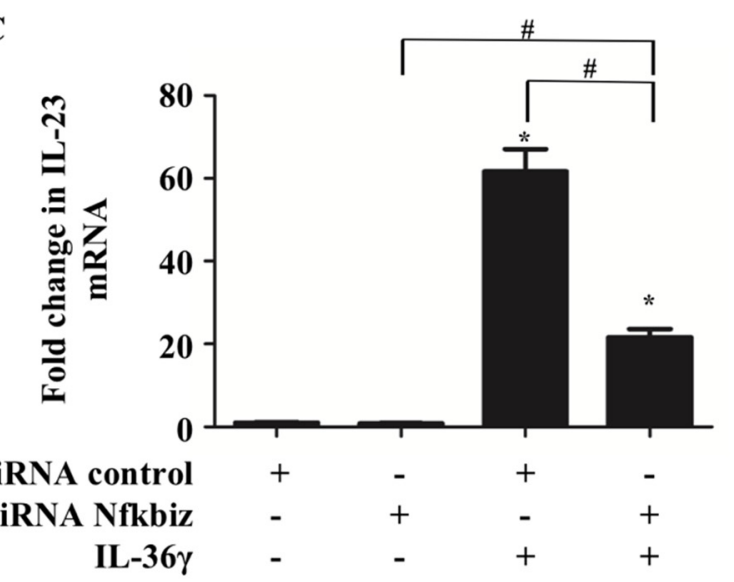

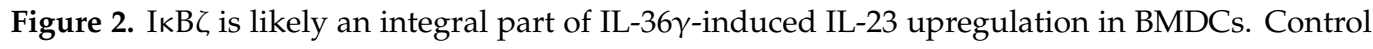
small interfering RNA (siRNA)- or Nfkbiz siRNA-transfected BMDCs were treated with/without IL-36 $(10 \mathrm{ng} / \mathrm{mL})$ for $1 \mathrm{~h}$ and analyzed via quantitative reverse transcription (qRT)-PCR and Western 
blotting. + / - indicates whether siRNA or IL-36 $\gamma$ is utilized. (A) qRT-PCR. (B) Western blotting. (C) qRT-PCR. (A,C) Data are expressed as the mean \pm standard error of the mean (SEM); $n=3$ /group. * Significant difference versus the control siRNA-transfected group with no IL-36 $\gamma$ stimulation $(p<0.05)$. \# Significant difference between the Nfkbiz siRNA-transfected and control siRNA-transfected groups that were stimulated with IL-36 $\gamma(p<0.05)$. mRNA levels normalized to Ywhaz mRNA expression are expressed as the fold change versus that in the control group. (B) I $\mathrm{B} \zeta$ expression was evaluated using anti-murine $\mathrm{I} \kappa \mathrm{B} \zeta$ antibody. Data are representative of experiments repeated three times with similar results.

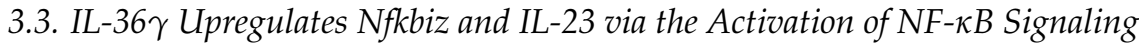

Next, we examined the mechanism by which IL-36 $\gamma$ upregulates Nfkbiz expression in murine BMDCs. Considering that IL-36 binding to the IL-36 receptor complex leads to the recruitment of MyD88 and activation of NF- $\mathrm{KB}$ signaling [16] and that Nfkbiz expression is regulated by phosphorylation of p65, a component of the NF- $\mathrm{B}$ heterodimer [24], we hypothesized that IL-36 $\gamma$ modulated Nfkbiz expression via NF- $k$ B signaling in murine BMDCs. We analyzed p65 phosphorylation in murine BMDCs stimulated with IL-36 $\gamma$ (100 ng/mL) for 10, 20, 30, 40, or $60 \mathrm{~min}$ using Western blotting. We confirmed that p65 phosphorylation was induced after $10 \mathrm{~min}$ of IL-36 $\gamma$ stimulation (Figure 3A). We further examined whether BAY 11-7082, an inhibitor of p65 phosphorylation, affects the upregulation of Nfkbiz induced by IL-36 $\gamma$ stimulation. We stimulated murine BMDCs with IL-36 $\gamma(100 \mathrm{ng} / \mathrm{mL})$ for $1 \mathrm{~h}$ in the absence or presence of BAY 11-7082 $(10,50$, or $100 \mu \mathrm{M}$ ) and measured Nfkbiz mRNA and IkB $\zeta$ protein expression by qRT-PCR (Figure 3B) and Western blotting (Figure 3C), respectively. BAY 11-7082 treatment inhibited Nfkbiz upregulation in a concentration-dependent manner (Figure 3B,C). Moreover, we examined whether BAY 11-7082 treatment inhibits the upregulation of IL-23 induced by IL-36 $\gamma$. We measured IL-23 production in the culture supernatant of BMDCs stimulated with IL-36 $\gamma$ $(100 \mathrm{ng} / \mathrm{mL})$ for $24 \mathrm{~h}$ in the absence or presence of BAY 11-7082 $(10,50$, or $100 \mu \mathrm{M}) \mathrm{using}$ ELISA. BAY 11-7082 treatment also inhibited the upregulation of IL-23 induced by IL-36 $\gamma$ stimulation in a concentration-dependent manner (Figure 3D).

\subsection{Metformin Treatment Inhibited the Upregulation of Nfkbiz and IL-23 Induced by IL-36 Stimulation by Impairing NF- $\mathrm{B}$ Signaling}

It has been reported that metformin controls NF- $\kappa B$ signaling [25]. Moreover, clinical studies of patients with psoriasis and T2DM have suggested that metformin administration may attenuate the disease activity of psoriasis $[19,20]$. Therefore, we hypothesized that metformin treatment affects the IL-36 $\gamma$-induced upregulation of Nfkbiz and IL-23 by modulating NF- $\mathrm{B}$ signaling in murine BMDCs. To test this, we analyzed p65 phosphory-

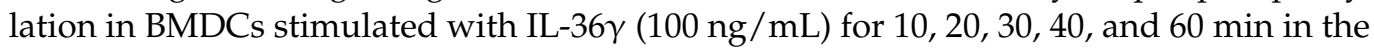
absence or presence of metformin $(5 \mathrm{mM})$ using Western blotting. Metformin treatment inhibited p65 phosphorylation induced by IL-36 $\gamma$ (Figure 4A). In addition, we evaluated

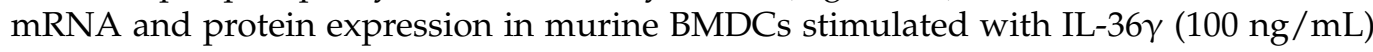
for $1 \mathrm{~h}$ in the absence or presence of metformin $(0.5,1$, or $5 \mathrm{mM})$. Metformin treatment inhibited the IL-36 $\gamma$-induced upregulation of Nfkbiz mRNA and I $\kappa \mathrm{B} \zeta$, protein expression in a concentration-dependent manner in BMDCs (Figure 4B,C). Subsequently, metformin treatment was revealed to inhibit the upregulation of IL-23 mRNA expression in BMDCs stimulated with IL-36 $\gamma$ for $1 \mathrm{~h}$ in a concentration-dependent manner (Figure 4D). In addition, metformin treatment downregulated IL-23 production in the culture supernatant of BMDCs stimulated with IL-36 $\gamma$ for $24 \mathrm{~h}$ in a concentration-dependent manner (Figure 4E). 
A

(kDa)

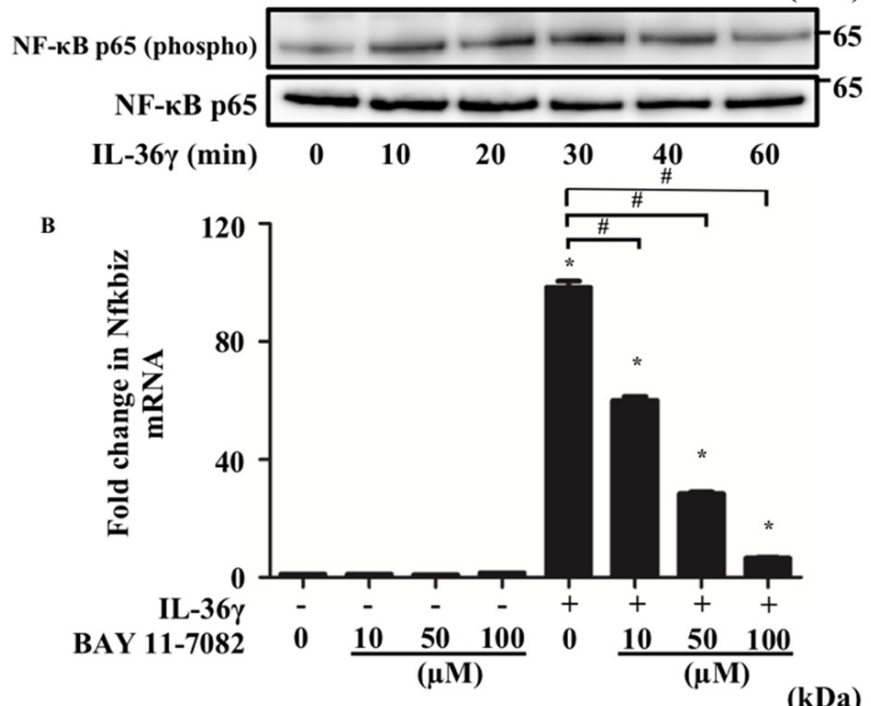

C

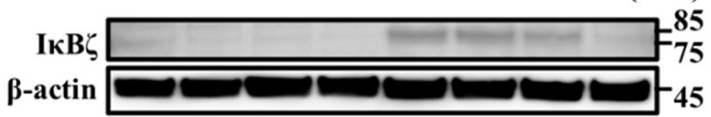

IL-36 $\gamma$

BAY 11-7082

) 10

\begin{tabular}{ccc}
- & - & - \\
10 & 50 & 100 \\
\hline
\end{tabular}

$(\mu \mathrm{M})$

D

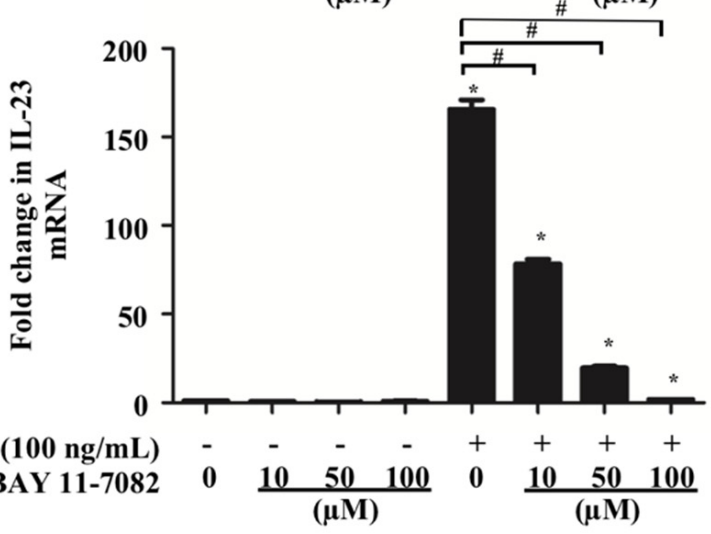

Figure 3. Nfkbiz expression was regulated by p65 phosphorylation in BMDCs. BMDCs were stimulated with IL-36 $\gamma(100 \mathrm{ng} / \mathrm{mL})$ for 10, 20, 30, 40, or $60 \mathrm{~min}$ (A). (A) Western blotting. BMDCs were stimulated with/without IL-36 $\gamma(100 \mathrm{ng} / \mathrm{mL})$ for $1 \mathrm{~h}(+/-)$ in the absence or presence of BAY 11-7082 (10, 50, or $100 \mu \mathrm{M})($ B-D). (B,D) Quantitative reverse transcription-PCR. (C) Western blotting. (B,D) Data are expressed as the mean \pm standard error of the mean; $n=3$ /group. ${ }^{*}$ Significant difference between the IL-36 $\gamma$-stimulated and control groups $(p<0.05)$. \# Significant difference

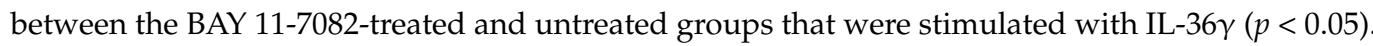
mRNA levels normalized to Ywhaz mRNA expression were expressed as the fold change versus that in the control group. $(\mathbf{A}, \mathbf{C})$ Data are representative of experiments repeated three times with similar results. + / - indicates whether IL-36 $\gamma$ is utilized. 


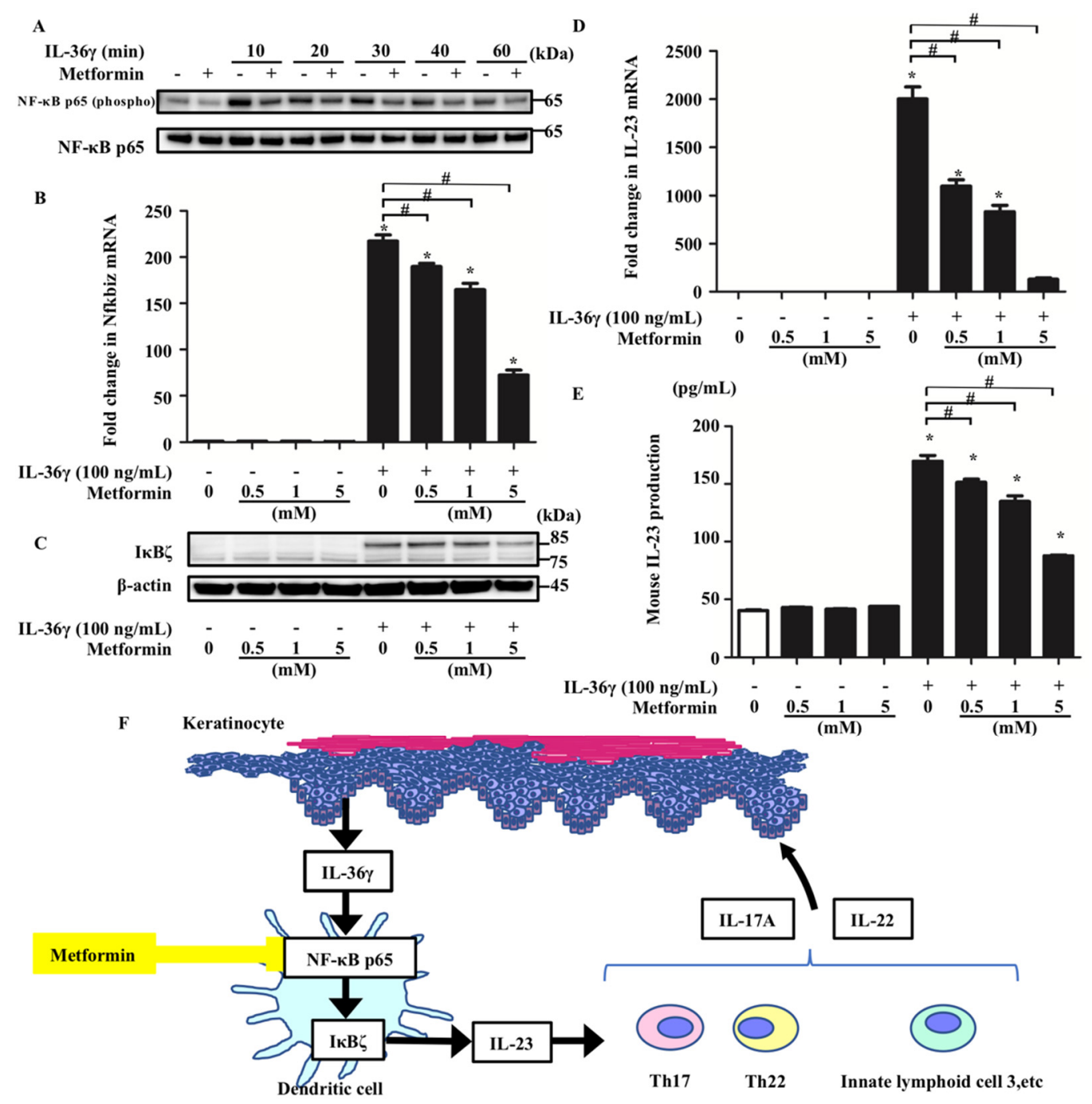

Figure 4. Metformin treatment inhibited IL-36 $\gamma$-induced upregulation of Nfkbiz and IL-23 by modulating NF- $\mathrm{B} B$ signaling

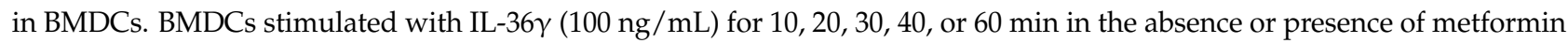

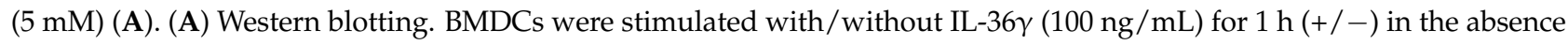
or presence of metformin $(0.5,1$, or $5 \mathrm{mM})$. (B,D,E). (B,D) quantitative reverse transcription-PCR. (E) ELISA. (F) Graphical abstract. Data are expressed as the mean \pm standard error of the mean; $n=3$ /group. * Significant difference between the IL-36 $\gamma$-stimulated and control groups $(p<0.05)$. \# Significant differences between the metformin-treated and control groups that were stimulated with IL-36 $\gamma(p<0.05)$. mRNA levels normalized for Ywhaz expression were expressed as fold changes versus that in the control group. $(\mathbf{A}, \mathbf{C})$ Data are representative of experiments repeated three times with similar results. $+/$ - indicates whether IL-36 $\gamma$ or metformin is utilized.

\section{Discussion}

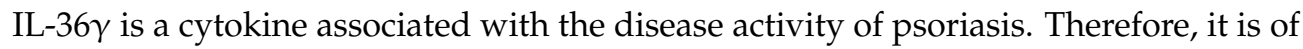
great interest to identify a strategy that inhibits the responses of downstream inflammatory cytokines. In this study, we obtained evidence that IL-36 $\gamma$ induces Nfkbiz upregulation, which subsequently leads to IL-23 upregulation, in murine BMDCs. Furthermore, we revealed that metformin treatment inhibited IL-23 upregulation via the impairment of Nfkbiz upregulation. To our knowledge, this is the first study providing evidence that metformin can modulate IL-36 $\gamma$-mediated IL-23 production in DCs, thereby contributing to the prevention of psoriasis. However, the systemic expression of IL-23 following the administration of IL-23 minicircle DNA [26] or transgenic expression of IL-23 derived from keratinocytes [27] can reportedly promote the development of psoriasis. Therefore, the 
production of IL-23 by cells other than DCs has also been reported to contribute to the pathogenesis of psoriasis, which is a limitation of this study.

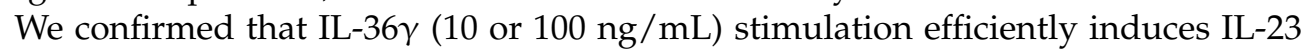
mRNA upregulation in murine BMDCs, which is consistent with previous findings [28,29] Furthermore, we revealed that IL-36 $\gamma$ stimulation increases IL-23 protein expression using ELISA. Although the concentration of $100 \mathrm{ng} / \mathrm{mL}$ is rather high, we considered it reasonable in this experiment system because IL-36 $\gamma$ is activated by neutrophil-derived proteases such as proteinase-3 [30].

$\mathrm{I} \kappa \mathrm{B} \zeta$, a transcriptional regulator of selective NF- $\mathrm{kB}$ target genes, has been identified as a crucial mediator of IL-36-driven psoriasis-related gene expression in keratinocytes [21];

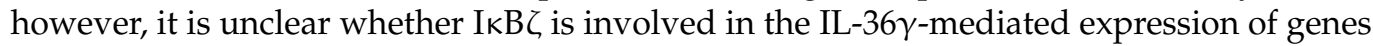
including IL-23 in DCs. Based on the result that depletion of Nfkbiz mRNA expression partially downregulated IL-23 mRNA expression induced by IL-36 stimulation, I $\mathrm{KB} \zeta$ is likely an integral part of IL-36-induced IL-23 upregulation in murine BMDCs. As we utilized siRNA transfection to deplete Nfkbiz mRNA expression, the low depletion efficiency may have resulted in weak repression of IL-23 mRNA expression.

Although several studies suggested that the molecular machinery underlying the regulation of $\mathrm{I} \kappa \mathrm{B} \zeta$ could be cell type-specific, it has been reported that the induction of $\mathrm{I} \kappa \mathrm{B} \zeta$ by IL-36 $\gamma$ is mediated by MyD88, NF- $\mathrm{kB}$, and STAT3 in human keratinocytes $[13,14]$. We found that inhibition of NF- $\mathrm{KB}$ activity by BAY 11-7082 treatment significantly downregu-

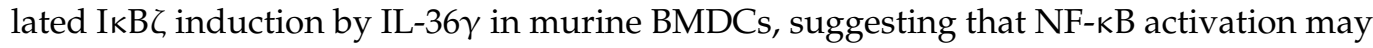

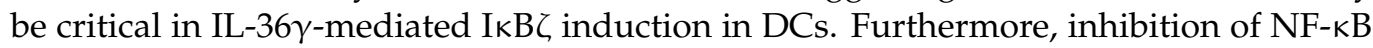
activity by metformin treatment had the same effect as BAY 11-7082 treatment. These data support the potential benefits of metformin treatment in patients with type 2 diabetes mellitus (T2DM) and psoriasis. As several studies revealed an association between T2DM and psoriasis $[31,32]$ and suggested a severity-dependent relationship between psoriasis and T2DM [17], the management of T2DM is considered extremely important in the treatment of psoriasis. Metformin treatment has been reported to inhibit several signaling pathways including the mammalian target of rapamycin [33] and mitogen-activated protein kinase signaling [34], in addition to NF- $\mathrm{kB}$ signaling. As such, we cannot thus exclude the possibility that other kinases might have been affected by metformin in the experiments. Further studies will be required to clarify this possibility.

We revealed here that metformin exerts anti-inflammatory effects on DCs, at least in part via pathways involving the inhibition of $\mathrm{I} \kappa \mathrm{B} \zeta$ production (Figure $4 \mathrm{~F}$ ). Our study presents the novel concept that pharmacological modulation by metformin of IL-36 $\gamma$ induced IL-23 production via I $\kappa B \zeta$ inhibition may offer a potential therapeutic approach to psoriasis. It can be hypothesized that oral metformin administration suppresses psoriasis by this mechanism. However, the clinical relevance of IL-23 inhibition by metformin requires further investigation.

Supplementary Materials: The following are available online at https://www.mdpi.com/article/ 10.3390/jcm10235610/s1, Table S1: Murine TaqMan gene expression assay probes.

Author Contributions: T.M.-T. and G.T. designed the experiments. T.M.-T., G.T., M.F. and T.N. wrote the manuscript. G.T., A.H.-H., T.M.-T., A.T.-Y. and M.T. performed the experiments. T.M.-T., G.T. and M.F. analyzed the results. All authors contributed to the interpretation of the research. All authors have read and agreed to the published version of the manuscript.

Funding: This research was funded by grants from the Ministry of Health, Labour and Welfare of Japan (R3-Shokuhin-Shitei-005) and JSPS KAKENHI (grant number 20K08653).

Institutional Review Board Statement: The study was conducted according to the guidelines of the Declaration of Helsinki and approved by the Institutional Review Board of KYUSHU UNIVERSITY (A21-283-0 and 1 June 2021).

Conflicts of Interest: The authors declare no conflict of interest. 


\section{References}

1. Michalek, I.M.; Loring, B.; John, S.M. A systematic review of worldwide epidemiology of psoriasis. J. Eur. Acad. Dermatol. Venereol. 2017, 31, 205-212. [CrossRef]

2. Furue, M.; Furue, K.; Tsuji, G.; Nakahara, T. Interleukin-17A and Keratinocytes in Psoriasis. Int. J. Mol. Sci. 2020, 21, 1275. [CrossRef] [PubMed]

3. Boehncke, W.H.; Schön, M.P. Psoriasis. Lancet 2015, 386, 983-994. [CrossRef]

4. Yan, B.; Liu, N.; Li, J.; Li, J.; Zhu, W.; Kuang, Y.; Chen, X.; Peng, C. The role of Langerhans cells in epidermal homeostasis and pathogenesis of psoriasis. J. Cell Mol. Med. 2020, 24, 11646-11655. [CrossRef] [PubMed]

5. Hawkes, J.E.; Yan, B.Y.; Chan, T.C.; Krueger, J.G. Discovery of the IL-23/IL-17 Signaling Pathway and the Treatment of Psoriasis. J. Immunol. 2018, 201, 1605-1613. [CrossRef]

6. Wohn, C.; Ober-Blöbaum, J.L.; Haak, S.; Pantelyushin, S.; Cheong, C.; Zahner, S.P.; Onderwater, S.; Kant, M.; Weighardt, H.; Holzmann, B.; et al. Langerin(neg) conventional dendritic cells produce IL-23 to drive psoriatic plaque formation in mice. Proc. Natl. Acad. Sci. USA 2013, 110, 10723-10728. [CrossRef]

7. Riol-Blanco, L.; Ordovas-Montanes, J.; Perro, M.; Naval, E.; Thiriot, A.; Alvarez, D.; Paust, S.; Wood, J.N.; von Andrian, U.H. Nociceptive sensory neurons drive interleukin-23-mediated psoriasiform skin inflammation. Nature 2014, 510, 157-161. [CrossRef]

8. Lee, E.; Trepicchio, W.L.; Oestreicher, J.L.; Pittman, D.; Wang, F.; Chamian, F.; Dhodapkar, M.; Krueger, J.G. Increased expression of interleukin 23 p19 and p40 in lesional skin of patients with psoriasis vulgaris. J. Exp. Med. 2004, 199, 125-130. [CrossRef]

9. Di Cesare, A.; Di Meglio, P.; Nestle, F.O. The IL-23/Th17 axis in the immunopathogenesis of psoriasis. J. Investig. Dermatol. 2009, 129, 1339-1350. [CrossRef]

10. Cai, Y.; Shen, X.; Ding, C.; Qi, C.; Li, K.; Li, X.; Jala, V.R.; Zhang, H.G.; Wang, T.; Zheng, J.; et al. Pivotal role of dermal IL-17-producing gammadelta T cells in skin inflammation. Immunity 2011, 35, 596-610. [CrossRef]

11. Gordon, K.B.; Duffin, K.C.; Bissonnette, R.; Prinz, J.C.; Wasfi, Y.; Reich, K.; Li, S.; Shen, Y.-K.; Szapary, P.; Randazzo, B.; et al. A Phase 2 Trial of Guselkumab versus Adalimumab for Plaque Psoriasis. N. Engl. J. Med. 2015, 373, 136-144. [CrossRef] [PubMed]

12. Papp, K.A.; Blauvelt, A.; Bukhalo, M.; Gooderham, M.; Krueger, J.G.; Lacour, J.-P.; Menter, A.; Philipp, S.; Sofen, H.; Tyring, S.; et al. Risankizumab versus Ustekinumab for Moderate-to-Severe Plaque Psoriasis. N. Engl. J. Med. 2017, 376, 1551-1560. [CrossRef] [PubMed]

13. Reich, K.; Warren, R.B.; Iversen, L.; Puig, L.; Pau-Charles, I.; Igarashi, A.; Ohtsuki, M.; Falqués, M.; Harmut, M.; Rozzo, S.; et al. Long-term efficacy and safety of tildrakizumab for moderate-to-severe psoriasis: Pooled analyses of two randomized phase III clinical trials (reSURFACE 1 and reSURFACE 2) through 148 weeks. Br. J. Dermatol. 2020, 182, 605-617. [CrossRef] [PubMed]

14. D’Erme, A.M.; Wilsmann-Theis, D.; Wagenpfeil, J.; Hölzel, M.; Ferring-Schmitt, S.; Sternberg, S.; Wittmann, M.; Peters, B.; Bosio,

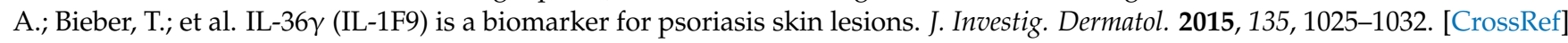
[PubMed]

15. Mercurio, L.; Morelli, M.; Scarponi, C.; Eisenmesser, E.Z.; Doti, N.; Pagnanelli, G.; Gubinelli, E.; Mazzanti, C.; Cavani, A.; Ruvo, M.; et al. IL-38 has an anti-inflammatory action in psoriasis and its expression correlates with disease severity and therapeutic response to anti-IL-17A treatment. Cell Death Dis. 2018, 9, 1104. [CrossRef]

16. Walsh, P.T.; Fallon, P.G. The emergence of the IL-36 cytokine family as novel targets for inflammatory diseases. Ann. N. Y. Acad. Sci. 2018, 1417, 23-34. [CrossRef]

17. Gelfand, J.M.; Wan, M.T. Psoriasis: A novel risk factor for type 2 diabetes. Lancet Diabetes Endocrinol. 2018, 6, 919-921. [CrossRef]

18. Brauchli, Y.B.; Jick, S.S.; Curtin, F.; Meier, C.R. Association between use of thiazolidinediones or other oral antidiabetics and psoriasis: A population based case-control study. J. Am. Acad. Dermatol. 2008, 58, 421-429. [CrossRef]

19. Singh, S.; Bhansali, A. Randomized placebo control study of insulin sensitizers (Metformin and Pioglitazone) in psoriasis patients with metabolic syndrome (Topical Treatment Cohort). BMC Dermatol. 2016, 16, 12. [CrossRef]

20. Singh, S.; Bhansali, A. Randomized placebo control study of metformin in psoriasis patients with metabolic syndrome (Systemic Treatment Cohort). Indian J. Endocrinol. Metab. 2017, 21, 581-587. [CrossRef]

21. Müller, A.; Hennig, A.; Lorscheid, S.; Grondona, P.; Schulze-Osthoff, K.; Kramer, D. IkB乙 is a key transcriptional regulator of IL-36-driven psoriasis-related gene expression in keratinocytes. Proc. Natl. Acad. Sci. USA 2018, 115, 10088-10093. [CrossRef]

22. Ovesen, S.K.; Schulze-Osthoff, K.; Iversen, L.; Johansen, C. IkB $\zeta$ is a Key Regulator of Tumour Necrosis Factor-a and Interleukin17A-mediated Induction of Interleukin-36g in Human Keratinocytes. Acta. Derm. Venereol. 2021, 101, adv00386. [CrossRef]

23. Siegert, I.; Schatz, V.; Prechtel, A.T.; Steinkasserer, A.; Bogdan, C.; Jantsch, J. Electroporation of siRNA into mouse bone marrow-derived macrophages and dendritic cells. Methods Mol. Biol. 2014, 1121, 111-119. [CrossRef] [PubMed]

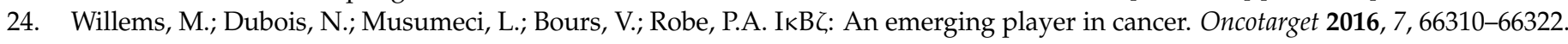
[CrossRef]

25. Sultuybek, G.K.; Soydas, T.; Yenmis, G. NF-кB as the mediator of metformin's effect on ageing and ageing-related diseases. Clin. Exp. Pharmacol. Physiol. 2019, 46, 413-422. [CrossRef] [PubMed]

26. Sherlock, J.P.; Joyce-Shaikh, B.; Turner, S.P.; Chao, C.-C.; Sathe, M.; Grein, J.; Gorman, D.M.; Bowman, E.P.; McClanahan, T.K.; Yearley, J.H.; et al. IL-23 induces spondyloarthropathy by acting on ROR- $\gamma \mathrm{t}^{+} \mathrm{CD}^{+} \mathrm{CD} 4^{-} \mathrm{CD} 8^{-}$entheseal resident $\mathrm{T}$ cells. Nat. Med. 2011, 18, 1069-1076. [CrossRef] [PubMed]

27. Chen, L.; Deshpande, M.; Grisotto, M.; Smaldini, P.; Garcia, R.; He, Z.; Gulko, P.S.; Lira, S.A.; Furtado, G.C. Skin expression of IL-23 drives the development of psoriasis and psoriatic arthritis in mice. Sci. Rep. 2020, 10, 8259. [CrossRef] [PubMed] 
28. Vigne, S.; Palmer, G.; Lamacchia, C.; Martin, P.; Talabot-Ayer, D.; Rodriguez, E.; Ronchi, F.; Sallusto, F.; Dinh, H.; Sims, J.E.; et al. IL-36R ligands are potent regulators of dendritic and T cells. Blood 2011, 118, 5813-5823. [CrossRef] [PubMed]

29. Kovach, M.A.; Singer, B.; Martinez-Colon, G.; Newstead, M.W.; Zeng, X.; Mancuso, P.; Moore, T.A.; Kunkel, S.L.; Peters-

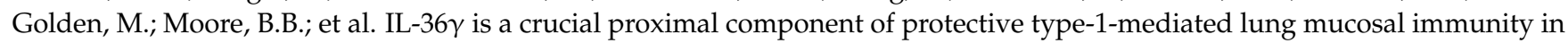
Gram-positive and -negative bacterial pneumonia. Mucosal Immunol. 2017, 10, 1320-1334. [CrossRef]

30. Henry, C.M.; Sullivan, G.P.; Clancy, D.M.; Afonina, I.S.; Kulms, D.; Martin, S.J. Neutrophil-Derived Proteases Escalate Inflammation through Activation of IL-36 Family Cytokines. Cell Rep. 2016, 14, 708-722. [CrossRef]

31. Yeung, H.; Takeshita, J.; Mehta, N.N.; Kimmel, S.E.; Ogdie, A.; Margolis, D.J.; Shin, D.B.; Attor, R.; Troxel, A.B.; Gelfand, J.M. Psoriasis severity and the prevalence of major medical comorbidity: A population-based study. JAMA Dermatol. 2013, 149, 1173-1179. [CrossRef] [PubMed]

32. Wan, M.T.; Shin, D.B.; Hubbard, R.A.; Noe, M.H.; Mehta, N.N.; Gelfand, J.M. Psoriasis and the risk of diabetes: A prospective population-based cohort study. J. Am. Acad. Dermatol. 2018, 78, 315-322. [CrossRef]

33. Nyambuya, T.M.; Dludla, P.V.; Mxinwa, V.; Mokgalaboni, K.; Ngcobo, S.R.; Nkambule, B.B. The impact of metformin and aspirin on T-cell mediated inflammation: A systematic review of in vitro and in vivo findings. Life Sci. 2020, 255, 117854. [CrossRef] [PubMed]

34. Kim, J.; Kwak, H.J.; Cha, J.Y.; Jeong, Y.S.; Rhee, S.D.; Kim, K.R.; Cheon, H.G. Metformin suppresses lipopolysaccharide (LPS)induced inflammatory response in murine macrophages via activating transcription factor-3 (ATF-3) induction. J. Biol. Chem. 2014, 289, 23246-23255. [CrossRef] [PubMed] 